\title{
Correction: Enhancement in the kV Portal Image Contrast Using Depth Normalization for Accurate Patient Localization
}

\author{
[J. Korean Phys. Soc. 72, 539 (2018)]
}

DOI: $10.3938 / j k p s .72 .539$

\author{
Hosang JeOn, Hanbean Youn, Jiho NAM, ${ }^{*}$ Jayoung LeE and Juhye LeE \\ Department of Radiation Oncology and Research Institute for Convergence of Biomedical Science and Technology, \\ Pusan National University Yangsan Hospital, Yangsan 50612, Korea \\ Dahl PARK, Wontaek KIM, Yongkan KI and Donghyun KIM \\ Department of Radiation Oncology, Pusan National University Hospital, Busan 46241, Korea \\ Ho Kyung KIM \\ School of Mechanical Engineering and Center for Advanced Medical \\ Engineering Research, Pusan National University, Busan 46241, Korea
}

DOI: $10.3938 /$ jkps. 75.749

The affiliation of Yongkan Ki should be corrected as follow:

Yongkan KI

Department of Radiation Oncology, Pusan National University School of Medicine, Busan 46241, Korea

*E-mail: jihonam@hanmail.net 